\title{
Outcome of Carotid Endarterectomy in a Tertiary Center of Nepal: A Retrospective Study
}

\author{
Prashiddha B Kadel, Uttam K Shrestha, Kajan R Shrestha, Dinesh Gurung
}

\author{
Author(s) affiliation \\ Department of Cardiothoracic and \\ Vascular Surgery, Maharajgunj \\ Medical Campus, Manmohan \\ Cardiothoracic and Vascular \\ Transplant Center, Institute of \\ Medicine, Maharajgunj, Kathmandu, \\ Nepal
}

\section{Corresponding author}

Prashiddha B Kadel, MBBS, MS prashiddhakadel@gmail.com

\section{Submitted}

Dec 2, 2020

\section{Accepted}

Mar 18, 2021

\begin{abstract}
Introduction

Carotid endarterectomy for carotid artery disease is one of the surgeries performed by vascular surgeons for carotid artery disease. The objective of this study is to describe the early and late outcome of the patient undergoing carotid endarterectomy and the association between the complication and comorbidities present previously in the patient.
\end{abstract}

\section{Methods}

All patients undergoing carotid endarterectomy at Manmohan Cardiothoracic Vascular and Transplant Centre between April 2010 to April 2020 were included. The follow-up data for upto a year from medical and clinical records, telephone interview regarding the immediate and late postoperative complications in patients with and without comorbidities were investigated and compared.

\section{Results}

The total study population was 42 patients. Two patients (4.7\%) developed stroke, one immediately in postoperative period and the other during follow up. There were two deaths (4.7\%) postoperatively due to cardiac events and three (7.14\%) recurrences of carotid stenosis among whom one (2.5\%) developed late stroke. Twenty six patients (61.90\%) were symptomatic prior to the procedure of which 20 patients (47.61\%) had brain infarct. Overall one year survival was $95.2 \%$ post procedure and overall complication rate was $7.14 \%$. The consequence in the form of death and stroke occurred more in the patients with comorbidities (3vs1) p=0.42.

\section{Conclusion}

The immediate and late postoperative complications following carotid endarterectomy were death $(4.7 \%)$, stroke $(4.7 \%)$, cranial nerve injury (9.5\%). The most frequent cause of death was postoperative cardiac event. Though major complications occurred more frequently in patients having comorbidities, it was statistically insignificant.

\section{Keywords}

Carotid artery, cerebrovascular disease, endarterectomy 


\section{INTRODUCTION}

$(2$ arotid artery disease as a disease entity is known since the start of civilization. In 1658, Wepfer highlighted the association of right sided hemiplegia with occlusion of left internal artery with inference of carotid artery disease and hemiplegia. In 1665, Willis described a case with asymptomatic carotid artery stenosis. ${ }^{1}$ During $20^{\text {th }}$ century, there was a concept that carotid artery bifurcation stenosis could lead to stroke due to clot embolism but the characteristics of stenosing lesion and the type of plaque causing embolism was described only after 50 years. ${ }^{2}$

The first surgery which decreased the incidence of stroke and other complications related to the atheroma was carotid endarterectomy and it was performed in women presenting with amaurosis fugax resulting in successful treatment. ${ }^{2,3}$

The European Carotid Surgery Trial proposed that in symptomatic patients, carotid endarterectomy provided improved stroke free interval in patients having greater than 70 percent stenosis. ${ }^{4}$ Similarly, the North American Symptomatic Carotid Trial also proposed that either symptomatic or asymptomatic patients with 70 percent or more stenosis, more benefitted from surgery with proportion of stenosis related to outcome of surgery. ${ }^{5}$ The Veterans Administration Asymptomatic Trial entails that symptomatic patients with history of frequent transient ischemic attacks or small cerebral infarction had favourable outcome after surgery as compared to medical therapy. ${ }^{6}$ The Asymptomatic Carotid Atherosclerosis Study showed that patients with $60 \%$ or more stenosis were candidate for carotid endarterectomy and had a lower risk of stroke at 5 years as compared to medical therapy. ${ }^{7}$

The main advantage of surgery is the high rate of freedom from stroke though there is associated risk of surgical complications. However, with the advent of refinement of surgical techniques and intrra operative monitoring, the complication rate has decreased significantly over the years. ${ }^{8}$

The objective of this study was to describe the early and late outcomes of patients that underwent carotid endarterectomy and study the association between complications and comorbidities present previously in the patient.

\section{METHODS}

This is a retrospective study conducted at Manmohan Cardiothoracic Vascular and Transplant Centre, Kathmandu, Nepal using medical records and telephone interview of the patients undergoing carotid endarterectomy in Manmohan Cardiothoracic Vascular and Transplant Centre. A total of 42 patients with carotid artery stenosis were enrolled in this study. The patients were referred by either physicians, cardiologist, neurologist and vascular surgeons. Comorbidities like hypertension, diabetes mellitus, chronic obstructive pulmonary disease (COPD), asthma were considered as variables in the study. Both symptomatic and asymtomatic patients with $\geq 70$ percent carotid artery stenosis on the basis of carotid Doppler ultrasound, underwent carotid angiography for proper delineation of anatomy of carotid vessels. There was slight difference in extend of stenosis observed between carotid Doppler ultrasound and carotid angiography. With stenosis of $\geq 70$ percent found on coronary angiography, both symptomatic and asymptomatic patients underwent carotid endarterectomy after proper evaluation of cardiac function and other baseline investigations. All baseline investigations were sent preoperatively and then surgery was planned and performed.

Any complications after surgery were noted. Postoperatively, all patients were started on oral antiplatelet therapy (dual antiplatelet therapy). All patients were then followed up in the OPD or via telephone. Patients with stroke or hemiparesis were then followed up with CT scan or MRI head and the size of infarct were noted and neurology consultation was done. Any death or loss to follow up were noted. All patients undergoing carotid endarterectomy underwent CT angiography postoperatively after 10-11 months if carotid artery duplex showed greater than $50 \%$ stenosis of operated carotid artery.

Carotid artery duplex scan was routinely carried out 6 months after surgery. Stenosis of more than $50 \%$ after initial surgery was marked as recurrence and interventions in the form of repeat carotid endarterectomy or carotid artery stenting was advised.

Fisher's Exact Test was applied at a significance level of $5 \%$. Survival rate was made according to the actuarial method. The SPSS program version 25 was used for the construction of tables and evaluation of data.

\section{RESULTS}

A total of 42 patients with carotid artery stenosis underwent carotid endarterectomy and the outcome was studied. In our study, there were 16 patients $\leq 60$ years while 26 patients $>60$ years with mean age of $64.3 \pm 18.7$ years. A total of 10 patients (23.8\%) were female and 32 patients(76.2\%) were male. In all, $57.1 \%$ had comorbidities of which 10 patients $(23.8 \%)$ had diabetes mellitus, 8 patients (19\%) had both hypertension and diabetes,6 patients(14.3\%) had only hypertension. Unilateral stenosis was present in $85.7 \%$ whereas $14.3 \%$ had bilateral disease at presentation. Sixteen patients had concomitant disease in the form of coronary artery disease or peripheral vascular disease of 
Table 1. Different symptoms of carotid artery disease

\begin{tabular}{lc}
\hline \multicolumn{1}{c}{ Symptoms } & Number \\
\hline Stroke within 6 months & 20 \\
Transient ischemic attack & 4 \\
Amaurosis fugax & 2 \\
\hline
\end{tabular}

which 12 patients (28.57\%) were diagnosed to have significant carotid artery disease after routine investigation of carotid artery using Doppler ultrasound as a part of coronary artery bypass graft preoperative investigation, whereas 4 patients $(9.5 \%)$ undergoing femoropopliteal or aortofemoral bypass had their carotid artery disease diagnosed while performing carotid doppler as a preoperative investigation. All patients having peripheral vascular disease underwent femoropopliteal or aortofemoral bypass after treatment of carotid artery stenosis.

Twenty six patients (61.9\%) were symptomatic (previous stroke, transient ischemic attack or amaurosis fugax (Table 1) whereas 16 patients (38.1\%) were asymptomatic in the form of no prior history of previous stroke, transient ischemic attack or amaurosis fugax. Carotid endarterectomy with great saphenous vein patch closure was done in 22 cases $(52 \%)$ which was on the discretion of the surgeon.
Table 2. Different complications encountered after carotid endarterectomy

\begin{tabular}{lc}
\hline \multicolumn{1}{c}{ Complications } & Number \\
\hline Massive bleeding & 2 \\
Early stroke & 1 \\
Death in post-operative period & 2 \\
Cranial nerve injury & 4 \\
Hypertensive crisis & 4 \\
Late stroke & 1 \\
Recurrence & 3 \\
\hline
\end{tabular}

Complications encountered during and after carotid endarterectomy have been shown in table 2. Massive bleeding requiring cell salvage support autotransfusion was seen in 2 cases (4.7\%) and 1 patient had stroke in the immediate postoperative period. There were 2 deaths (4.7\%) in the post operative period, the cause of death being cardiac event, 4 patients $(9.5 \%)$ had cranial nerve injury (hypoglossal nerve being the most common) in the immediate postoperative period which recovered with time. Postoperative hypertensive crisis occured in 4 cases $(9.5 \%)$. Late stroke occurred in 1 patient during 1 year follow up. Three patients had recurrence of the disease in the form of re-stenosis of more than $50 \%$, these patients were offered for redo endarterectomy but they refused, 2 patients underwent carotid artery stenting as an alternative

Table 3. Distribution of postoperative death and stroke by age, sex, stump pressure, comorbidities and after the use of shunt in the patients undergoing carotid endarterectomy

\begin{tabular}{|c|c|c|c|c|}
\hline \multirow{2}{*}{ Characteristics } & \multicolumn{2}{|c|}{ Death or stroke } & \multirow{2}{*}{ Total } & \multirow{2}{*}{$p$-value } \\
\hline & No & Yes & & \\
\hline \multicolumn{5}{|l|}{ Age } \\
\hline$\leq 60$ yrs & $16(38 \%)$ & 0 & $16(38 \%)$ & 0.13 \\
\hline$>60$ yrs & $22(52.5 \%)$ & $4(9.5 \%)$ & $26(62 \%)$ & \\
\hline \multicolumn{5}{|l|}{ Sex } \\
\hline Male & $28(66.6 \%)$ & $4(9.5 \%)$ & $32(76.2 \%)$ & 0.32 \\
\hline Female & $10(23.8 \%)$ & 0 & $10(23.8 \%)$ & \\
\hline \multicolumn{5}{|c|}{ Comorbidities (HTN/DM) } \\
\hline Yes & $21(50 \%)$ & $3(7 \%)$ & $24(57.2 \%)$ & 0.42 \\
\hline No & 17 (40.5\%) & $1(2.4 \%)$ & $18(42.8 \%)$ & \\
\hline \multicolumn{5}{|l|}{ Symptoms } \\
\hline Yes & $24(57.2 \%)$ & $2(4.7 \%)$ & $26(61.9 \%)$ & 0.49 \\
\hline No & $14(33.33 \%)$ & $2(4.7 \%)$ & $16(38.1 \%)$ & \\
\hline \multicolumn{5}{|l|}{ Carotid stump pressure } \\
\hline >50mmhg & $32(76.2 \%)$ & $2(4.7 \%)$ & 34 (80.9) & 0.16 \\
\hline$<50 \mathrm{mmhg}$ & $6(14.2)$ & $2(4.7 \%)$ & $8(19.1 \%)$ & \\
\hline \multicolumn{5}{|l|}{ Shunt } \\
\hline Yes & $2(4.7 \%)$ & $2(4.7 \%)$ & $4(9.5 \%)$ & 0.12 \\
\hline No & $36(85.7 \%)$ & $2(4.7 \%)$ & $38(90.5 \%)$ & \\
\hline
\end{tabular}


to surgery. Table 3 compares the distribution of death or stroke as a consequence of surgery on the basis of age, sex, stump pressure, comorbidities and use or disuse of shunt in the patients undergoing carotid endarterectomy. The difference in outcome in terms of stroke or death were not statistically significant between the groups based on age, sex, stump pressure, comorbidities and use of shunt as shown in table 3.

\section{DISCUSSION}

Carotid endarterectomy though considered a relatively safe surgery is associated with early and late complications. ${ }^{9}$ The cut-off of carotid artery stenosis was kept at 70 percent and included both symptomatic and asymptomatic population. Different multicentric trials signify that patients having $70 \%$ stenosis or more should be operated. Asymptomatic Carotid Atherosclerosis Study states that all patients withstanding surgery with stenosis of greater than $60 \%$ should be operated ${ }^{5,10}$, in the end it all depends on the discretion of surgeons whether to operate or not at around $60 \%$ stenosis with symptoms and should be judged on case to case basis.

Carotid artery disease is a part of multisystem disease with peripheral artery disease, coronary artery disease. According to this study, peripheral artery disease was found in 4 cases $(9.5 \%)$ and 12 cases $(28 \%)$ had coronary artery disease. Similar to the study done by Yan Wu et al which showed that carotid atherosclerosis was an independent risk factor for coronary heart disease [OR $=2.66,95 \%$ confidence interval $(95 \% \mathrm{Cl}), 2.05-$ 3.46, $p<0.001] .{ }^{11}$ Klop et al in their study showed that carotid artery disease with $\geq 75 \%$ stenosis were present in 62 patients(14.9\%) with peripheral artery disease signifying the fact that carotid artery screening should always be considered in a patient of peripheral artery disease. ${ }^{12}$

In this study, wide range of patients with age ranging from 45 to 86 years age with mean age of $64.31 \pm 8.7$ years underwent carotid endarterectomy with no significant difference in complications related to different age group. Here we compared between patients of age $\leq 60$ years and $>60$ years ( $n=16$ vs 26$)$ or $(38 \%$ vs $62 \%)$, though patients of age less than 60years had more bleeding (2vs1), the major adverse complications were more common in age group more than 60 years with death in 2 patients $(4.5 \%)$, postoperative hypertensive crisis in 4 patients (all occurring in patients with age greater than 60yrs),cranial nerve injury in 4 patients (age greater than 60yrs). However it was found to be statistically insignificant $(p=0.66)$. There were 2 cases of stroke with 1 in immediate postoperative period and another late stroke in patients, both occurred in the patients with age $>60$ years $(p=0.134)$ which is similar to the study done by Jeong $\mathrm{MJ}$ et al which concluded that age is an independent risk factor for major adverse events following CEA within 4 years. ${ }^{13}$ Summarizing, major complications occurred more frequently in the age group $\geq 60$ years.

After carotid Doppler study, all patients underwent CT angiogram (CTA) of the carotid vessels for proper delineation of the extent of stenosis and feasibility of the surgery. In a study done by Titi M et al, comparison of Doppler ultrasound and CT angiography in evaluation of carotid stenosis they found that the decision of operative management changed in $28 / 187$ cases (16\%) (95\% Cl 0.11-0.21) on the basis of carotid angiography. ${ }^{14}$

In cases of bilateral carotid artery stenosis, some surgeons take a gap of at least 15 days between the sequential surgery. In this study, sequential surgery of the bilateral carotid artery was not done though 4 patients had bilateral disease. Primary endarterectomy was performed on the artery with more severe stenosis or that associated with symptoms, the latter artery with less significant stenosis was to be operated only after 2-3 weeks. However these patients opted for carotid artery stenting after primary endarterectomy.

Carotid artery shunt is commonly done in our institute if the stump pressure is below $50 \mathrm{mmHg}$ which has been found to decrease post operative stroke. In our study, carotid shunt was used in 4 patients $(9.5 \%)$, among whom 2 patients developed major complication (stroke or death). In 38 patients $(90.47 \%)$ where shunt was not required intraoperatively, 2 patients had stroke or death concluding that $50 \%$ patient undergoing carotid endarterectomy using shunt had major complication whereas $5.2 \%$ patient with stump pressure greater than $50 \mathrm{~mm} \mathrm{Hg}$ where shunt was not required had adverse complications in the form of death or stroke.However, it was not found to be significant statistically ( $p=0.12)$. In a study done by Kwaan et al in evaluation of stump pressure as a guide during carotid endarterectomy, the study concluded that carotid shunt doesnot ensure cerebral perfusion rather the wakefulness during surgery serves to prevent stroke when surgery is performed under local anesthesia. ${ }^{15}$

Patients with hypertension and diabetes as comorbidities had more postoperative stroke and death as compared to patients without comorbidities $(n=3$ Vs $n=1) \quad p=0.42$. Similarly, patients with comorbidities had more postoperative hospital stay (5.25 days VS 5.22 days). Summarizing, patient with comorbidities had higher frequency of complication as compared to patients without comorbidities though statistically insignificant. In a research done by Jeong et al, the outcomes between diabetics and nondiabetics were compared, and discovered 
that diabetics were at greater risk of late major adverse event and identified diabetes as an independent risk factor for occurrence of stroke. ${ }^{16}$ In the present study major complications occurred more frequently in patients with comorbidities. The overall complication rate in the study is $7 \%$ which is slightly more than the criteria set by Guidelines for carotid endarterectomy of the Special Writing Group of the Stroke Council and American Heart Association which has $6 \%$ of postoperative stroke and death, if one has to demonstrate its superiority as compared to medically treated symptomatic carotid stenosis patient. 5,7 The slight increase in postoperative stroke and death might be due to the involvement of both symptomatic and asymptomatic patients with carotid artery stenosis.

In this study, the incision used was a longitudinal which spares the greater auricular nerve in comparison to the transverse incision. Following surgery cranial nerve injury (unilateral hypoglossal nerve, recurrent laryngeal nerve) was present in 4 patients $(9.5 \%)$ and the most common cranial nerve injury was hypoglossal nerve followed by recurrent laryngeal nerve however the cranial nerve deficit recovered over months and there was no residual deficit which is similar to the study done by Cunningham which showed that the most common cranial nerve injury was hypoglossal (27) followed by, recurrent laryngeal(17) and emphasized that the risk of nerve injury persisting beyond hospital discharge was around $4 \%$ after carotid endarterectomy $195 \%$ $\mathrm{Cl}$ 2.9-4.7) which decreased with increasing experience. ${ }^{17}$

After completion of endarterectomy, patch closure technique was used whenever surgeons felt the caliber of artery small, 22 patients (52\%) had patch closure out of which 2 patients (9.09\%) had recurrence $(p=0.53)$. While the study done by Clagett demonstrated the routine saphenous vein patch closure did not yield superior results and was also found to have a greater incidence of early recurrence( $12.9 \%$ vs $1.7 \%$ with $p<0.05)$ and post operative time however if anatomical risk factors was present for recurrent disease saphenous vein patch closure seemed to be appropriate. ${ }^{18}$

Focussing on the long term complication, one patient had a stroke with debilitation with overall survival in our study being $95.2 \%$ at one year.

\section{CONCLUSION}

The immediate and late postoperative complication following carotid endarterectomy was death $(4.7 \%)$, stroke $(4.7 \%)$, cranial nerve injury $(9.5 \%)$. The most frequent cause of death was postoperative cardiac events. Though major complications occurred more frequently in patients having comorbidities, it was statistically insignificant.

\section{CONFLICT OF INTEREST}

None declared.

\section{REFERENCES}

1. Aguiar ET, Lederman A, Higutchi $C$ et al. Early and late results of carotid endarterectomy: retrospective study of 70 operations. Sao Paulo Med J. 2001;119(6):206-11.

2. Salles $L R$, Puech-Leão $P$, Netto $B M$ et al. Risk factors of stroke in carotid endarterectomy. Revistado Hospital das Clinicas. 1997:52(6):291-4.

3. Eastcott HH, Pickering GW, Rob CG et al. Reconstruction of internal carotid artery in a patient with intermittent attacks of hemiplegia. The Lancet.1954;264(6846):994-6.

4. European Carotid Surgery Trialists' Collaborative Group. MRC European Carotid Surgery Trial: interim results for symptomatic patients with severe (70-99\%) or with mild (0-29\%) carotid stenosis. lancet. 1991;337(8752):1235-43.

5. North American Symptomatic Carotid Endarterectomy Trial Collaborators*. Beneficial effect of carotid endarterectomy in symptomatic patients with high-grade carotid stenosis. N Engl J Med. 1991;325(7):445-53

6. Hobson RW, Weiss DG, Fields WS et al. Veterans Affairs Cooperative Study Group. Efficacy of carotid endarterectomy for asymptomatic carotid stenosis. N Engl J Med. 1993;328(4):221-7.

7. Moore WS, Young B, Baker WH et al. Howard VJ, ACAS Investigators. Surgical results: a justification of the surgeon selection process for the ACAS trial. J Vasc Surg. 1996;23(2):323-8.

8. Biller J, Feinberg WM, Castaldo JE et al. Guidelines for carotid endarterectomy: a statement for healthcare professionals from a Special Writing Group of the Stroke Council, American Heart Association. Stroke. 1998;29(2):554-62

9. Müller MD, Lyrer P, Brown MM et al. Carotid artery stenting versus endarterectomy for treatment of carotid artery stenosis. Cochrane Database of Systematic Reviews. 2020(2).

10. Jeong MJ, Kwon $\mathrm{H}_{\text {, Jung }} \mathrm{CH}$ et al. Comparison of outcomes after carotid endarterectomy between type 2 diabetic and non-diabetic patients with significant carotid stenosis. Cardiovasc Diabetol. 2019;18(1):41

11. Wu Y, He J, Sun $X$ et al. Carotid atherosclerosis and its relationship to coronary heart disease and stroke risk in patients with type 2 diabetes mellitus. Medicine. 2017;96(39).

12. Klop RB, Eikelboom BC, Taks AC et al. Screening of the internal carotid arteries in patients with peripheral vascular disease by colour-flow duplex scanning. Eur J Vasc Surg. 1991;5(1):41-5.

13. Jeong MJ, Kwon SU, Kim MJ et al. Effects of patient age on outcomes after carotid endarterectomy: A retrospective, singlecenter study in Korea. Medicine. 2019;98(32).

14. Titi M, George C, Bhattacharya D et al. Comparison of carotid Doppler ultrasound and computerised tomographic angiography in the evaluation of carotid artery stenosis. Surgeon. 2007;5(3):132-6.

15. Kwaan JH, Peterson GJ, Connolly JE et al. Stump pressure: an unreliable guide for shunting during carotid endarterectomy. Arch Surg. 1980;115(9):1083-5.

16. Jeong MJ, Kwon $\mathrm{H}$, Jung $\mathrm{CH}$ et al. Comparison of outcomes after carotid endarterectomy between type 2 diabetic and non-diabetic patients with significant carotid stenosis. Cardiovasc Diabetol. 2019 Dec 1;18(1):41.

17. Cunningham EJ, Bond R, Mayberg MR et al. Risk of persistent cranial nerve injury after carotid endarterectomy. J Neurosurg. 2004;101(3):445-8.

18. Clagett GP, Patterson CB, Fisher Jr DF et al. Vein patch versus primary closure for carotid endarterectomy: a randomized prospective study in a selected group of patients. J Vasc Surg. 1989;9(2):213-23. 